\title{
CAT: A tool for eliciting knowledge on how to perform procedures
}

\author{
KENT E. WILLIAMS and ELLIOTT HULTMAN \\ University of Central Florida, Orlando, Florida \\ and \\ ARTHUR C. GRAESSER \\ University of Memphis, Memphis, Tennessee
}

\begin{abstract}
Procedural tasks involve context-sensitive sequences of actions that are performed in pursuit of goals. Procedural knowledge specifies how to do something (e.g., repairing a car) but not how the physical system works (e.g., how the engine works). We have developed a computer program that elicits procedural knowledge from individuals with varying amounts of domain knowledge (ranging from novices to experts) and varying amounts of computer literacy. The tool is called CAT (cognitive analysis tool). CAT is an extension of a class of cognitive models known as GOMS, which stands for goals, operators, methods, and selection rules. The tool guides the user in articulating the goals (and subgoals) the user wants to accomplish, the operators (actions, steps) to accomplish each goal, the alternative methods of accomplishing goals, the conditions in which each method is applied, and exceptional circumstances when goals are suspended and restarted. CAT can be used on most IBMcompatible microcomputers.
\end{abstract}

One form of knowledge consists of the cognitive and behavioral procedures that agents perform in pursuit of goals. Examples of procedural knowledge range from mundane routine activities, such as driving a car and baking a cake, to tasks that require high expertise, such as using a sophisticated computer program and tactical planning. Procedural knowledge specifies the steps that an agent performs to achieve a goal, but it does not necessarily capture the deep knowledge about the physical system that explains why the procedures are successful. The procedure for changing the oil in a car would delineate the various steps, actions, and decisions of the mechanic, but not how the engine works. Thus, procedural knowledge represents "how to do it" knowledge but not "how it works" knowledge (Kieras \& Bovair, 1984).

There is a long history in the cognitive sciences of attempting to understand and represent procedural knowledge (Lashley, 1951; Miller, Galanter, \& Pribram, 1960). Cognitive scientists have developed computer models that simulate the actions that are performed and the order of the actions when agents perform routine "scripted" activities (Schank \& Abelson, 1977), when they solve novel problems (Laird, Newell, \& Rosenbloom, 1987; Newell

This research was funded by the Office of Naval Research (N0001495-11113), under the direction of Susan Chipman. Requests for reprints should be sent to K. E. Williams, Performance Support Technologies, 3432 Mission Bay Blvd., Suite 280, Orlando, FL 32817, or to A. C. Graesser, Department of Psychology, University of Memphis, CAMPUS BOX 526400, Memphis, TN 38152-6400 (e-mail: a-graesser@, memphis.edu). lndividuals who are interested in acquiring the CAT software should write K. E. Williams at the above address or contact him at kwilliam@1eknowledge.com. The cost of the software is $\$ 50$ plus shipping and handling.
\& Simon, 1972), and when they interact with computers and devices (Card, Moran, \& Newell, 1983; Kieras, 1988; Zachary \& Ross, 1991). Some of these computational theories have been tested in psychology experiments. For example, the knowledge contained in scripts, plans, and other packages of procedural knowledge can, to some extent, predict what actions are recalled, the order of recall, and memory intrusions (Bower, Black, \& Turner, 1979; Graesser, 1978). The EPIC model developed by Meyer and Kieras (1997) is able to predict response times and subtle patterns of timing during the execution of strategically driven motor skills. The computational model developed by Card et al. (1983), called GOMS (which will be discussed shortly), generates detailed predictions about human performance when adults interact with computers and devices. GOMS has been able to account for human performance when adults interact with text editors (Card et al., 1983), spreadsheets (Olson \& Nilsen, 1988), video games (John, Vera, \& Newell, 1990), telephone systems (Gray, John, \& Atwood, 1993), and medical programs (Beard, Smith, \& Denelsbeck, 1996). Thus, procedural knowledge has been investigated in rich detail, both computationally and empirically.

\section{PROPERTIES OF PROCEDURAL KNOWLEDGE}

Procedural knowledge has a number of properties that are widely acknowledged and that would be incorporated in any serious theory (Card et al., 1983; Miller et al., 1960; Newell \& Simon, 1972). We will enumerate nine properties in this section, but it is beyond the scope of this article to cover the precise formal specifications and the psy- 
chological evidence for these properties. We will hereafter refer to cognitive procedures, which consist of the cognitive and behavioral actions that a person performs when the person attempts to achieve a primary goal.

Procedural knowledge and CAT will be discussed in the context of an easy cognitive task with which adults are quite familiar: driving a car. The advantage of selecting this familiar task is that it is in the common ground of virtually everyone in this culture, and the reader will not get sidetracked into trying to understand an unfamiliar technical procedure. Driving a car also has all of the critical features of procedural knowledge, even though much of this knowledge has been automatized from extensive practice. When a person drives a car, there are cognitive actions that include decisions, perceptual searches, and memory retrieval, in addition to behavioral actions that involve movements of the fingers, hand, foot, and head. We acknowledge, however, that the primary value of CAT lies in dissecting and understanding unfamiliar cognitive procedures and tasks. More will be said about the uses of CAT later in this article.

\section{Cognitive Procedures Versus Rigid Sequences}

Most cognitive procedures are not simply rigid chains of actions or associative chains of stimulus-response units. Instead, the set of actions and the order of actions vary across situations in a fashion that is sensitive to circumstances in the world. For example, suppose that the driver of an automobile is very hot, which triggers the goal of "decreasing the temperature in the car." The driver does not automatically generate the following invariant sequence of behaviors: (a) move right hand to $\mathrm{A} / \mathrm{C}$ on/off switch, (b) place index finger on on/off switch, (c) press on/off switch, (d) move hand to temperature control, (e) place index finger and thumb on temperature control, (f) move fingers to right (to increase cool air).

All of these steps would be executed in some situations, whereas some steps would be skipped in others. If the $\mathrm{A} / \mathrm{C}$ unit is not on, then all of the above steps would be executed (sequence abcdef). If the $\mathrm{A} / \mathrm{C}$ unit was already on, then the first three steps would be skipped, and the sequence would be def. If the hand was already near the $\mathrm{A} / \mathrm{C}$ temperature control and the $\mathrm{A} / \mathrm{C}$ unit was on, then the sequence would be ef. If the $\mathrm{A} / \mathrm{C}$ temperature was already adjusted correctly but the $\mathrm{A} / \mathrm{C}$ unit was not on, then the sequence would be abc. The output of a cognitive procedure is a flexible construction that is sensitive to a dynamic world, not a rigid behavior chain.

\section{Cognitive Procedures Are Goal Directed}

The actions in a cognitive procedure are constructed in an effort to achieve a goal. The main goal in the above example is to decrease the temperature in the car. There frequently are auxiliary goals when a cognitive procedure is generated, such as "moving the car forward" and "avoiding collisions" in the above example. Thus, multiple goals normally constrain the actions in a cognitive procedure.

\section{Goal Hierarchies}

A goal hierarchy is constructed whenever a method is selected to achieve the main goal. Again consider the previous example in which the main goal is to "decrease temperature in the car" (Node 1). The subgoals of Node 1 would be "turn on $\mathrm{A} / \mathrm{C}$ unit" (Node 1.1) and "get $\mathrm{A} / \mathrm{C}$ unit to emit more cold air" (Node 1.2). Subgoal 1.1 is achieved by three primitive actions (abc in the above example), as is Subgoal 1.2 (def in the above example). In principle, there can be many levels in the goal hierarchy, but, in practice, there presumably are limits on the number of levels that the human mind can monitor. At some point at the lower level of a goal hierarchy, a subgoal or a step cannot be broken down further because the step is a primitive action (or what is called a primitive operator in Card et al.'s GOMS model). What constitutes a primitive action depends on the "grain size" that the researcher wants to adopt. "Turn on $\mathrm{A} / \mathrm{C}$ unit" may be regarded as a primitive action for a human factors engineer who wants to evaluate the design of the entire panel in an automobile, but that level would be too crude for an engineer who designs hand controls and switches.

\section{Order of Steps at One Level}

When one node is directly superordinate to a set of subordinate nodes, the subordinate "sibling" nodes are normally ordered, but not always. In the above example, Nodes $a, b$, and $c$ are sibling nodes that are directly subordinate to Node 1.1 ("turn on A/C switch"). The temporal order of Nodes $a, b$, and $c$ is fixed by virtue of physical necessity. That is, it is physically necessary to move the hand to the $\mathrm{A} / \mathrm{C}$ switch (Node a) in order to place the index finger on the switch (Node b), and Node $b$ is physically necessary for pressing the on/off switch (Node c). Physical necessity thus is one constraint that determines the ordering of sibling nodes. Another constraint is efficiency (i.e., order XY is more efficient than $\mathrm{YX}$ in terms of task completion time). For example, it is more efficient to get the $\mathrm{A} / \mathrm{C}$ unit to emit more cold air (Node X) before adjusting the air vents $(Y)$ than vice versa; the air vents would sometimes need to be readjusted in the YX ordering, resulting in steps YXY, whereas only two steps are needed in the $X Y$ ordering. Another constraint on ordering is convention, the mere habit or cultural preference for an order without any obvious advantage in efficiency or physical necessity. Nevertheless, sometimes steps are not ordered at all. Two steps may be needed to achieve a goal, but the ordering of steps is totally arbitrary (e.g., releasing the emergency brake and turning on the engine).

\section{Limited Number of Sibling Nodes}

The number of sibling nodes is normally limited to seven units. Thus, a goal directly dominates at most seven subgoals, and a subgoal directly dominates at most seven nodes. Researchers have periodically argued that the node limitation provides a foundation for chunking, optimizes search through long-term memory, and permits the 
scanning and comparison of nodes in a limited-capacity working memory (Graesser \& Mandler, 1978; Mandler, 1967; Newell \& Simon, 1972).

\section{Alternative Methods to Achieve a Goal \\ There normally is more than one method to achieve a goal. If one method does not succeed, then other meth- ods are tried until the goal is eventually achieved or the goal ends up being dropped. For example, there are a va- riety of methods for achieving the goal of decreasing the temperature in the car: opening the car window, turning on a fan, and turning on the air conditioner. When one fails, another one is tried.}

\section{Methods Are Context Sensitive}

A particular method is appropriate under a particular set of circumstances. Opening the car window is appropriate for achieving the example goal of decreasing the temperature. This method is particularly appropriate when the weather is cold and it is not raining. Turning on a fan is appropriate when the weather is cold and rainy. Turning on the air conditioner is appropriate when the weather is hot. Thus, the application of the methods is sensitive to the states of the world. Sometimes the "inappropriate" methods are attempted after the appropriate ones are exhausted.

\section{Suspension and Restarting of Goals}

A goal may be suspended under specific conditions and resumed later on when the conditions change in a dynamic world. For example, a passenger might grumble about being cold, so the driver suspends the goal of decreasing the temperature in the car. The goal resumes after the passenger leaves the vehicle.

\section{Goal Satisfaction}

When a goal is achieved, the agent stops applying methods to achieve the goal. It should be noted, however, that some goals are always operative (e.g., avoiding collisions).

The nine properties in this section do not exhaust the total set of properties that researchers have ascribed to procedural knowledge. Moreover, there is not total agreement that all of these properties are characteristic of procedural knowledge. However, most theories do embrace these properties. The knowledge elicitation tool that we developed incorporates these nine properties.

\section{THE GOMS MODEL}

Card et al. (1983) developed a computer model that simulates cognitive procedures. The model is called GOMS, an acronym for goals, operators, methods, and selection rules. As discussed in the previous section, there is a structured set of goals that direct a cognitive procedure. Each goal refers to a desire, objective, mission, aim, or purpose that an agent wants to achieve. Each goal is expressed in the form of a symbolic goal node, such as
(GOAL 1: driver wants to decrease temperature in car), (SUBGOAL 1.1: driver wants to turn on $\mathrm{A} / \mathrm{C}$ unit), and (SUBGOAL 1.2: driver wants to get the $\mathrm{A} / \mathrm{C}$ unit to emit more cold air).

The operators are the steps that accomplish a goal. The operators can be described at any level of detail. A high-level operator is a subgoal (i.e., subtask) that must be accomplished by lower level steps; for example, Goal 1 is achieved by the two steps expressed as Subgoals 1.1 and 1.2. A low-level operator is a primitive action, such as "move right hand to A/C on/off switch," "place index finger on on/off switch," press on/off switch," and so on. The GOMS model predicts the amount of time that it takes to execute these primitive behavioral operators on the basis of psychological laws and empirical results. For example, Fitts's (1954) law predicts that the amount of time it takes to move a finger and press a target button on the basis of the distance the finger moves and the size of the button. The primitive operators also include cognitive acts, such as moving the eyes to a display position and making a decision among a set of alternative options. Again, GOMS predicts the completion times for these primitive cognitive acts on the basis of psychological laws. According to Hick's (1952) law, decision times increase logarithmically as a function of the number of options and the base-rate likelihood of each option.

A method designates the operators that can be applied in order to achieve a goal. The method normally consists of an ordered sequence of operators (called steps), but sometimes the ordering is optional. Thus, Goal 1 is achieved by completing the two subgoals, with Subgoal 1.1 serving as Step 1 and Subgoal 1.2 serving as Step 2. In turn, Subgoal 1.1 is achieved by executing three steps with primitive operators, as expressed below:

(METHOD-1: driver cool car with air conditioner) (STEP-1 OPERATOR: subgoal 1.1: driver wants to turn on $\mathrm{A} / \mathrm{C}$ unit)

(STEP-2 OPERATOR: subgoal 1.2: driver wants the $\mathrm{A} / \mathrm{C}$ to emit more cold air)

(METHOD 1.1: driver turns on $\mathrm{A} / \mathrm{C}$ unit)

(STEP-1 OPERATOR: primitive action: driver moves right hand to $\mathrm{A} / \mathrm{C}$ on/off switch)

(STEP-2 OPERATOR: primitive action: driver places index finger on on/off switch)

(STEP-3 OPERATOR: primitive action: driver presses on/off switch)

There can be alternative methods for achieving a particular goal. For example, Methods 2 and 3 are other ways to achieve the goal of decreasing the temperature in the car: (METHOD-2: driver cool car with fan) and (METHOD3: driver open window). GOMS can simulate a task completion time for each of the methods. The task completion times are computed after considering the primitive operators that are performed and circumstances in the world that affect the completion times for the primitive operators. For example, the time to complete Method 1 
will depend on the size of the on/off switch and the distance between the driver's normal hand location and the location of the $\mathrm{A} / \mathrm{C}$ on/off switch.

The selection rules specify the conditions under which the alternative methods are applied to achieve the goal. The conditions refer to states of the world and cognitive states of the agent. GOMS uses "IF $<$ state $>$, THEN < action>" production rule formalism to express these contingencies, which is compatible with most symbolic models in the cognitive sciences (Anderson, 1993; Just \& Carpenter, 1992; Kieras, 1988; Laird et al., 1987). The following three production rules would capture the conditions under which each method is applied:

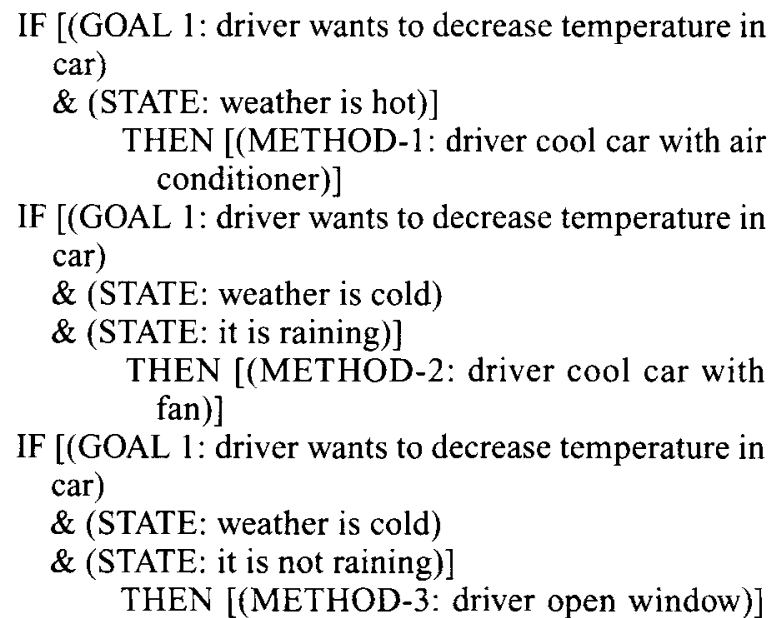

An agent may use multiple methods to achieve a goal if the ideal method does not succeed. For example, if the driver discovers that the window control is broken, the driver might use the air conditioner or the fan even though it is cold outside. Again, GOMS simulates the amount of time it takes to achieve a goal, given the states of the world, the methods that are applied, and the completion times of the primitive operators.

As discussed earlier, GOMS has already proven its ability to successfully predict human performance when cognitive procedures are executed. Most tests of GOMS have been in the area of human-computer interaction (Beard et al., 1996; Card et al., 1983; Kieras, 1988; Olson \& Nilsen, 1988). For example, Card et al. (1983) simulated the activities of experienced users of word processors at varying grain sizes (i.e., the keystroke level, subtasks completed, major tasks completed). GOMS successfully predicted what operators are performed, task completion times for the operators, and the ordering of operators. It was gratifying to learn that GOMS had some validity, given the amount of effort and time it took to develop the model. Given its validity, the utility of GOMS is enormous. GOMS can be used for forecasting the usability of existing artifacts, as well as alternative designs for new artifacts before they are manufactured.

Nevertheless, there are two limitations of GOMS that have prevented a widespread use in psychological research and applied projects. The first limitation is a prac- tical one. The development of a full-scale GOMS model takes a great deal of time, money, and expertise. The developers include computer programmers, experts who have mastered the cognitive procedures, knowledge engineers who extract the goals, operators, methods, and selection rules from topic experts, and human factors engineers. It takes a long time, measured in years, to organize and coordinate these experts. Such projects are therefore very expensive. Beard et al. (1996) have recently demonstrated that a small, scaled-down version of GOMS (which they call Quick and Dirty GOMS) can be a useful tool for solving specific practical problems, but their Quick and Dirty GOMS was not developed as a general-purpose tool.

The second limitation of GOMS is more theoretical. With rare exceptions, GOMS models have not implemented all of the properties of cognitive procedures that were discussed in the previous section. For example, most GOMS models do not handle the execution of multiple high-level goals, multiple tasks, and the suspension/restarting of goals. More recent models in the GOMS family, such as the COGNET system developed by Zachary and Ross (1991), have expanded the GOMS architecture by integrating it with a multiple, parallel, software agent architecture.

We have developed a computer tool that has the potential to expand GOMS modeling efforts to users with varied expertise: CAT (cognitive analysis tool). CAT assists users in describing GOMS-like cognitive procedures. The tool can incorporate all nine properties of cognitive procedures, including goal suspension and restarting. The tool guides users with a wide range of expertise in GOMS, domain knowledge about the procedures, and computer literacy. Therefore, the tool can be used by a person who has had a brief introduction to GOMS (e.g., a 5-page article), modest experience with the cognitive procedure, and a small amount of experience with computers (e.g., an occasional user of an IBMcompatible microcomputer with Microsoft Windows). Of course, CAT can also be used by computer hackers who have a large amount of experience with GOMS modeling and with the cognitive procedure being modeled. CAT should therefore facilitate more modeling efforts in the GOMS tradition. Given that Beard et al. (1996) have demonstrated that a Quick and Dirty GOMS can be useful in solving some real-world applications, the utility of CAT should be substantial when it is impractical to develop a full-scale GOMS model.

\section{CAT: A TOOL FOR EXTRACTING KNOWLEDGE ABOUT COGNITIVE PROCEDURES}

The purpose of CAT is to guide the user in extracting declarative knowledge about cognitive procedures. The user could be a domain expert on the cognitive procedure who uses the knowledge acquisition tool on himself or herself. The user could also use the tool to extract 
knowledge from another domain expert. CAT creates a GOMS-like node structure as the user creates and describes the content of the nodes that refer to goals, subgoals, methods, steps, operators, conditions, exceptions, goal suspensions, and so on. The node structure is automatically saved in a file as the user creates and modifies the nodes in the cognitive procedure.

\section{Hardware and Software Requirements}

CAT can be used on any IBM-compatible computer with a 386, 486, or Pentium processor and a minimum of 4.0 MB of RAM memory. The operating system is a Microsoft Windows Version 3.1 or a Microsoft Windows 95. It should be noted that CAT is not a front end to another system (such as GOMS, Soar, or ACT-R) but rather is a stand-alone tool in an IBM environment.

\section{Interface Features}

CAT has the normal features of a Windows environment, such as multiple menus and menu options, selection of options with a mouse and point-and-click facility, and a keyboard for alphanumeric input. There are some additional features that are not supplied by the Windows environment. The tool automatically saves whatever nodes the user has created or modified. There is an automatic help facility in addition to the normal extended help that is presented when selecting a "Help" option. For the automatic help, the interactive window has a context-sensitive help area at the bottom of the screen. This area displays information about the window's active control item that is currently under the pointing cursor. There are two modes of operation: a "guidance" mode and a "freeplay edit" mode. The guidance mode is geared for novice and intermediate users because it guides the user through the process of developing the cognitive procedure and the appropriate constraints on each node. This guidance mode prompts the user to supply the appropriate text input. The freeplay edit mode is geared for experienced users and for users who want to make changes to an existing procedural structure. This mode has a graphical user interface with "Edit" and "Navigation" options. There is a graphical tree structure for representing the goals, methods, actions, conditions, and so on. It should be noted that the example screens in the present article assume that the user is in the guidance mode, so these example screens involve text input. Finally, there is a comprehensive tutorial that can be accessed by selecting a "Tutorial" option.

\section{Creating a Goal}

The first node to be articulated is the main goal of the cognitive procedure. Figure 1 presents the screen display that prompts the user to specify the goal. There is a field for the name of the goal (e.g., "driver wants to decrease the temperature in the car") and for a lengthy description of the goal. After the goal is specified, the user can declare that the goal node be saved (the "OK" option) or be canceled (the "Cancel" option). Extended help is available by selecting the "Help" option. Context-sensitive help is automatically supplied in the region at the bottom of the screen.

\section{Creating the Steps and Operators in a Method}

A method consists of a sequence of operators. Each operator can be a primitive action or a subgoal. The order of the operators is designated by step numbers. Figure 2

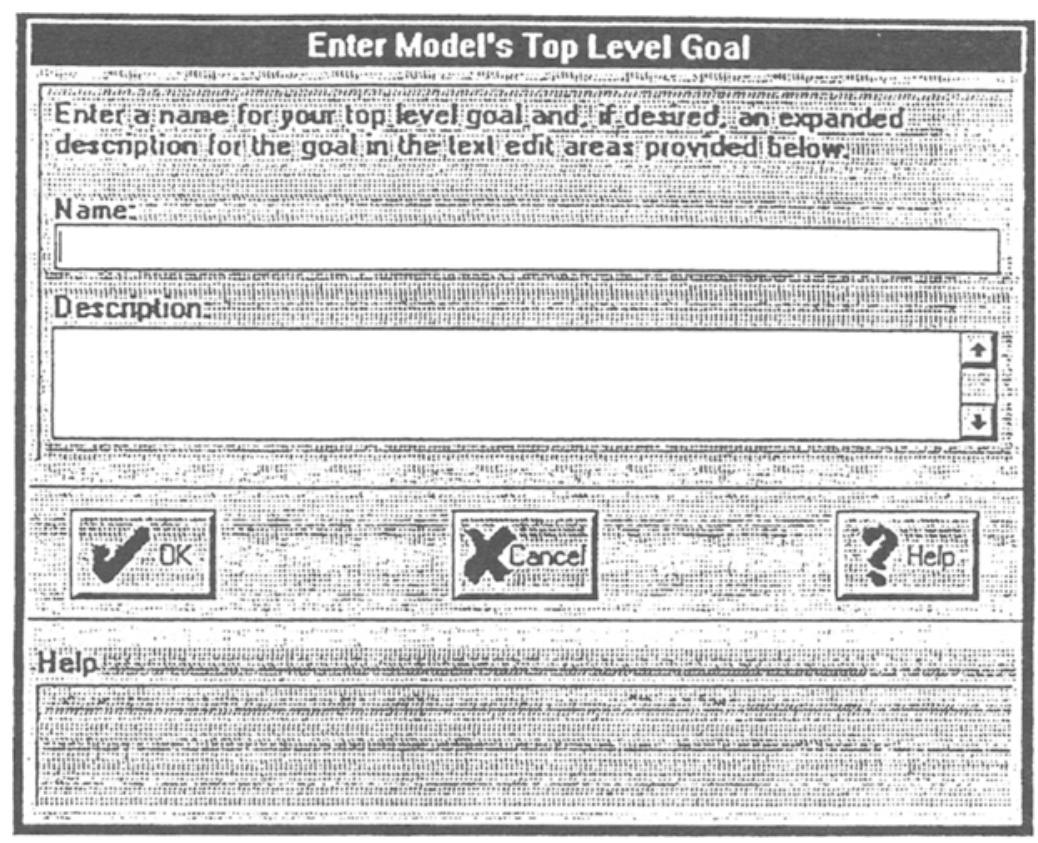

Figure 1. CAT screen that prompts the user to describe a goal. 


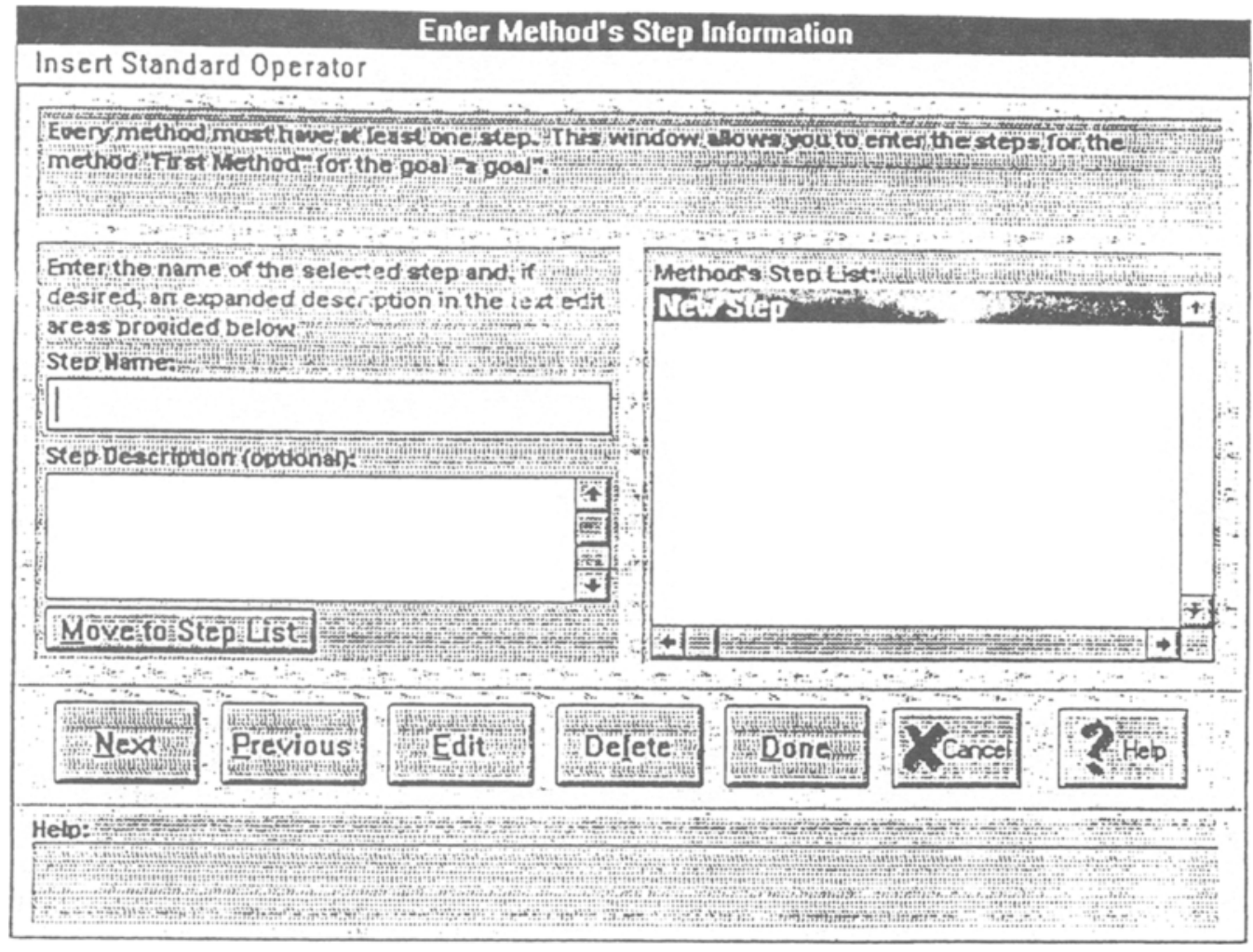

Figure 2. CAT screen that prompts the user to describe the steps of a method.

shows the screen that prompts the user to describe one of the steps in the top-level method. The user enters the step name and description in the middle-left region, whereas a list of all of the steps is displayed in the middle-right region. There is a default limit of seven steps associated with any given method. However, this default limit may be changed by the user. The "Next" and "Previous" options allow the user to move to subsequent steps and previous steps, respectively. The "Edit" option allows the user to edit the current step, and the "Delete" option deletes it. The user selects "Done" when all of the steps of the method are correct.

There are five types of operators associated with a step. A "simple" step designates either a subgoal or a primitive operator and is the most common type of step in most procedures. When the user declares this option, the computer presents a screen that is the same as Figure l except that the banner prints out "subgoal" instead of "goal." A "decision" step defines an "if-then-else" frame. That is, if a particular condition exists, then the operator is performed; if the condition does not exist, then another option is performed. This option permits a branching capability within a single step. When the user declares this option, there is a screen that prompts the user to supply an IF, THEN, and ELSE field. A "goto" step defines an unconditional branch to another step in the method. This is important when the procedure requires looping and recycling steps within a method. A "store" step stores up to five chunks of data into a specific working memory location, whereas the "recall" step fetches data from working memory. Therefore, CAT can implement models that have extensive interactions between procedural knowledge and working memory (Anderson, 1993; Just \& Carpenter, 1992; Kieras, 1988; Laird et al., 1987).

It is possible to reorder steps after the total set of steps are described for a method. After all of the steps have been described for a method, a screen queries the user about the intended order of the steps. There are three options available to the user: "As entered," "no specific order," and "another order." According to the first option, the order in which the steps are executed follows the original numerical order of the steps. There are no ordering constraints according to the second option. When the third option is selected, however, another screen is presented that guides the user in reordering the steps, and the new order is stored when the user selects a "Done" option.

CAT assumes that a particular method can contain only seven steps. As discussed earlier, this limitation reflects cognitive limitations on long-term memory, working memory, and consciousness. The constraint does not imply that there are only seven primitive operators subsumed under a method. Rather, there can be a hierarchical multileveled structure of subgoals that collectively end up dominating dozens of primitive operators. In 
order to provide for the hierarchical multileveled structure, a set of steps is sometimes consolidated into a single subgoal step. A screen is presented that guides the user in identifying what steps to consolidate, and another screen guides the user in labeling the subgoal.

\section{Creating Alternative Methods}

The proverbial fact that there is more than one way to skin a cat is one of the hallmarks of GOMS and CAT. After the user finishes describing one method to achieve a goal or subgoal, the user is queried whether there is another method. If the user selects the "Yes" option, he/she proceeds to numbering, labeling, and describing another method. However, as discussed earlier, the alternative methods are normally tuned to particular states in the world, as specified by the selection rules. There are screens that prompt the user to describe these conditions of the selection rule. As with most of the screens that prompt the user for descriptions, (1) the top region of the screen announces what sort of information is being supplied (in this case, creating the selection rule information), (2) the middle-left region prompts the user for a name and description of the condition, (3) the middleright region keeps track of the list of conditions, (4) the lower middle region has a set of options for searching, modifying, and saving the list of conditions, and (5) the bottom region presents context-sensitive help.

\section{Exceptions to a Cognitive Procedure}

There normally are exceptional conditions that require a successful agent to suspend a goal (or subgoal) or abandon the goal in favor of another goal. For example, a driver will suspend or abandon the goal of decreasing the temperature in a car if a passenger complains about being cold or if the driver gets too cold. The user is prompted to declare these exceptional conditions after the user has described all of the methods of a main goal. CAT guides the user in specifying the conditions of goal/subgoal suspension and exactly what goals and subgoals are suspended. In the case of goal suspension, CAT prompts the user to declare the conditions in which a goal is restarted. There can be several configurations of conditions that prompt the suspension or abandonment of goals.

\section{Failed Primitives and Resolving Impasses}

The last phase of constructing a cognitive procedure is to identify any steps that can fail during model execution and what to do when these failures occur. There may be times when a subgoal or a primitive operator may potentially fail. The user is prompted to declare what to do (i.e., what other goal to jump to) when each of the primitive operators fail. The user also has the option of specifying, for each subgoal, how to resolve an impasse by jumping to another goal.

\section{Executing a Cognitive Procedure}

Once a cognitive procedure has been described and saved, it can be executed in order to validate it. This is accomplished by selecting an "Execute Model" menu option. When this option is selected, the middle-left region of the screen displays the steps that are executed. Of course, the execution of some steps depends on the state of the world, as in the case of selection rules, exceptions, and alternative methods. The user is prompted to choose among alternative options that declare relevant states of the world, and the stream of steps subsequently ensues.

\section{USES OF CAT}

The most obvious use of CAT is for experts on technical topics who want to dissect the cognitive procedures in a technical domain. Some procedures reach a level of complexity where the expert might lose track of the methods, preconditions, consequences, and ordering of the steps. Indeed, a combinatorial explosion problem may emerge from a plan that has only a few dozen methods, a handful of structural levels, and a modest number of preconditions, consequences, and ordering constraints on each step (Hammond, Fasciano, Fu, \& Converse, 1996). The Office of Naval Research funded the development of CAT for the benefit of military tactical planning. A commander of a ship is sometimes faced with a situation in which there are multiple tactical plans and several possible consequences of each plan. If the commander has the luxury of thoughtful tactical planning, as opposed to quick decisions under stress, then CAT can be used to trace through the alternative plans and possible consequences. In a similar fashion, procedures can be dissected by experts in manufacturing, equipment design, management, operations research, and so on.

A less obvious use of CAT is for education. Suppose, for example, that the procedure for operating and maintaining a piece of equipment was implemented in CAT. A student could use this program to learn how to operate the equipment successfully, how to maintain it, and what happens when it is not properly used. Another educational application is in training students about cognitive modeling, cognitive procedures, and task analysis. CAT would serve as a "training wheels" system before students move on to GOMS, Soar, ACT-R, and similar sophisticated models of cognitive procedures.

\section{EXTENSIONS OF CAT}

CAT is currently being expanded in two fundamental ways in order to enhance the utility of GOMS-like modeling efforts. The first extension is to simulate execution times for achieving the main goal, given the states of the world that are declared by the user. Card et al. (1983) had theoretical estimates of the completion times for primitive behaviors and cognitive processes, based on psychological laws and available empirical research. Task completion times were also derived for completing subgoals and higher level chunks of operators. Using a similar approach, we have developed computer tools that 
simulate task completion times for single-step and multiple-step procedures (Graesser \& Marks, 1993; Meyer $\&$ Kieras, 1997). We are currently attempting to integrate these task completion time simulators with CAT.

The second extension is to expand the types of knowledge that can be incorporated in CAT. CAT described in this article focuses exclusively on the procedural "how to do it" knowledge. The expanded tool incorporates more elaborate knowledge structures with causal networks, spatial relations, taxonomic hierarchies, and other classes of conceptual structures (Graesser \& Clark, 1985; Lehmann, 1992). When these elaborated structures are created, the user assigns each node to a node category (e.g., concept, state, event, goal), and pairs of nodes are connected by categorized, directed relations (e.g., is-a, cause, reason, manner, has-as-parts, north-of). The strength of these connections are also estimated by the user. The more elaborated representations provide some foundation for tuning GOMS applications to world constraints and for building mental models of the domain knowledge.

\section{REFERENCES}

ANDERSON, J. R. (1993). Rules of the mind. Hillsdale, NJ: Erlbaum. Beard, D. V., Smith, D. K., \& Denelsbeck, K. M. (1996). Quick and dirty GOMS: A case study of computed tomography interpretation. Human-Computer Interaction, 11, 157-180.

Bower, G. H., Black, J. B., \& TuRner, T. J. (1979). Scripts in memory for text. Cognitive Psychology, 11, 177-220.

Card, S. K., Moran, T. P., \& Newell, A. (1983). The psychology of human-computer interaction. Hillsdale, NJ: Erlbaum.

FITTS, P. M. (1954). The information capacity of the human motor system in controlling the amplitude of movement. Journal of Experimental Psychology, 47, 381-391.

Graesser, A. C. (1978). How to catch a fish: The memory and representation of common procedures. Discourse Processes, 1, 72-89.

GRAESSER, A. C., \& CLARK, L. C. (1985). Structures and procedures of implicit knowledge. Norwood, NY: Ablex.

GraESSER, A. C., \& MANDLER, G. (1978). Limited processing capacity constrains the storage of unrelated sets of words and retrieval from natural categories. Journal of Experimental Psychology: Human Learning \& Memory, 4, 86-100.

Graesser, A. C. \& MARKs, W. (1993). Models that simulate driver performance with hand controls. In B. Peacock \& W. Karwowski (Eds.),
Automotive ergonomics: Human factors in the design and use of automobiles (pp. 383-399). London: Taylor \& Francis.

Gray, W. D., John, B. E., \& ATwoon, M. E. (1993). Project Ernestine: A validation of GOMS for prediction and explanation of real-world task performance. Human-Computer Interaction, 8, 209-237.

Hammond, K. J., Fasciano, M. J., Fu, D. D., \& Converse, T. (1996). Actualized intelligence: Case-based agency in practice. Applied Cognitive Psychology, 10, 73-83.

HICK, W. E. (1952). On the rate of gain of information. Quarterly Journal of Experimental Psychology, 4, 11-26.

John, B. E., Vera, A. H., \& Newell, A. (1990). Toward real-time GOMS (Tech. Rep. No. CMU-CS-90-195). Pittsburgh, PA: Carnegie Mellon University, School of Computer Science.

Just, M. A., \& CARPENTER, P. A. (1992). A capacity theory of comprehension: Individual differences in working memory. Psychological Review, 99, 122-149.

KIERAS, D. E. (1988). Towards a practical GOMS model methodology for user interface design. In M. Helander (Ed.), Handbook of humancomputer interaction (pp. 135-158). Amsterdam: North-Holland.

Kieras, D. E., \& BovaiR, S. (1984). The role of a mental model in learning to operate a device. Cognitive Science, 8, 255-274.

Laird, J. E., NeWell, A., \& Rosenbloom, P. S. (1987). SOAR: An architecture for general intelligence. Artificial Intelligence, 33, 1-64.

LASHLEY, K. (1951), The problem of serial order in behavior. In L. A. Jeffress (Ed.), Cerebral mechanisms in behavior. New York: Wiley.

LehmanN, F. (Ed.) (1992). Semantic networks in artificial intelligence. New York: Pergamon Press.

MandleR, G. (1967). Organization and memory. In K. W. Spence \& J. T. Spence (Eds.), The psychology of learning and motivation (Vol. 1, pp. 327-372). New York: Academic Press.

Meyer, D. E., \& Kieras, D. E. (1997). A computational theory of executive cognitive processes and multiple-task performance: Part 1. Basic mechanisms. Psychological Review, 104, 3-65.

Miller, G. A., Galanter, E., \& Pribram, K. H. (1960). Plans and the structure of behavior. New York: Holt, Rinehart \& Winston.

Newell, A., \& Simon, H. A. (1972). Human problem solving. Englewood Cliffs, NJ: Prentice-Hall.

OLSON, J. R., \& NilsEN. E. (1988). Analysis of cognition involved in spreadsheet software interaction. Human-Computer Interaction, $\mathbf{3}$, 309-350.

SchANK, R. C., \& ABELSON, R. (1977). Scripts, plans, goals, and understanding. Hillsdale, NJ: Erlbaum.

ZACHARY, W. W., \& Ross, L. (1991). Enhanced human-computer interaction through use of embedded COGNET models. In Proceedings of the Human Factors Society 35th Annual Meeting (pp. 425430). Santa Monica, CA: Human Factors Society.

(Manuscript received October, 17, 1996; revision accepted for publication May 7, 1997.) 